\title{
Search for Color Sextet Mesons in Early LHC Experiments
}

\section{Edmond L. BERGER* ${ }^{* \dagger}$}

Argonne National Laboratory

E-mail: bergerdanl.gov

The possible production of beyond-the-standard-model charge $4 / 3$ color-sextet mesons is explored at the Large Hadron Collider. They could be observed through their decay into a pair of same-sign top quarks. Final states are examined in which both top quarks decay semi-leptonically. The energy of the charged lepton from the top quark semi-leptonic decay serves as a good measure of the top quark polarization, which, in turn determines the quantum numbers of the mesons and distinguishes vectors from scalars.

35th International Conference of High Energy Physics

July 22-28, 2010

Paris, France

\footnotetext{
* Speaker.

${ }^{\dagger}$ The work reported here was done in collaboration with Qing-Hong Cao, Chuan-Ren Chen, Gabe Shaughnessy, and Hao Zhang. It was supported financially by the U. S. Department of Energy under Contract No. DE-AC02-06CH11357.
} 


\section{Introduction}

The cross section for production of top quarks is relatively high at the energies of the Large Hadron Collider (LHC). While conventional mechanisms produce either a single top quark or a top-antitop pair, it is important to be alert to the observation of a pair of same-sign top quarks. One consequence would be the appearance of a pair of same-sign leptons with large transverse momentum. In recent papers [1, 2], we explore the potential for discovery of exotic color-sextet scalar and vector mesons in the production of a pair of same-sign top quarks in early runs of the LHC at $7 \mathrm{TeV}$. The standard model (SM) backgrounds are small. We demonstrate that one can measure the meson masses and the top-quark polarization, and confirm the scalar or vector nature of the resonance with $1 \mathrm{fb}^{-1}$ of integrated luminosity.

Important in our analysis is the recognition that among the products of semi-leptonic top quark decay, the direction of the charged-lepton is highly correlated with the top quark spin. The top quark polarization can be measured from the distribution in the helicity angle $\theta$ between the chargedlepton momentum in the top-quark rest frame and the top-quark momentum in the center-of-mass frame of the production process. The $S U(2)_{L}$ gauge triplet scalars decay to $t_{L} t_{L}$, and the $S U(2)_{L}$ gauge singlet scalars to $t_{R} t_{R}$. Here, $t_{L}$ and $t_{R}$ denotes top quarks with left-handed and right-handed polarization. In either case, both top quarks produce the same angular distribution, either $(1-$ $\cos \theta) / 2\left(t_{L} t_{L}\right)$ or $(1+\cos \theta) / 2\left(t_{R} t_{R}\right)$, allowing unambiguous identification of the scalar [1], and the distinction between gauge triplet- and singlet-scalars. A $S U(3)_{C}$ color sextet vector decays into a $t_{L} t_{R}$ pair. In this case, the observed inclusive angular distribution in the final state would be a flat, a sum of the shapes from the $t_{L}$ and the $t_{R}$ decays. The flat profile would distinguish a vector from a scalar, but it also would admit a mechanism that yields unpolarized top quarks. In Ref.[2], we establish that one can separate the angular distributions corresponding to $t_{L}$ and $t_{R}$ from the color sextet vector decay into a $t_{L} t_{R}$ pair. The solution relies of the introduction of asymmetric cuts on the momenta of the leptons from the top-quark decays.

\section{Model Context and Calculation}

The model context in which we consider color sextet mesons and the pertinent Lagrangian are described in Refs. [1], 2] where references to previous and related work may also be found. We focus on direct channel production of the mesons via $u u$ scattering, exploiting the large $u u$ valence quark parton luminosity at LHC energies. The coupling of the sextet mesons to two uptype quarks is constrained by the measurement of $D^{0}-\bar{D}^{0}$ mixing which is affected by the sextet mesons at the tree level. Data on the mixing parameters yield a strong constraint on the product $g_{u u} g_{c c}$ of couplings to the up-type first and second generation quarks, allowing production of the sextet mesons at the LHC if the coupling $g_{c c}$ to the second generation quarks is minimized.

It is important to retain the top quark spin correlations in the calculation because the kinematic distributions of the decay products depend strongly on the top quark spin. The new sextet scalar meson interacts only with right-handed gauge singlet quarks so the top quarks from its decay are mainly right-handed. A charged lepton from a right-handed top quark decay has a harder momentum spectrum than one from a left-handed top quark. No available event generator calculates color sextet scalar and vector induced $t t$ production and subsequent $t$ decay with full top quark spin cor- 
relations included. We calculate the helicity amplitudes for $u u \rightarrow t t \rightarrow b W^{+}\left(\rightarrow \ell^{+} v\right) b W^{+}\left(\rightarrow \ell^{+} v\right)$ and implement the process in our own parton-level Monte Carlo code.

In considering sextet meson decay into same-sign top-top pairs $t$, we focus on the same sign dilepton decay mode in which the $W$ bosons from both $t \rightarrow W b$ decays lead to a final state containing an electron or muon (denoted $\ell$ ), $W \rightarrow \ell v$, accounting for about $5 \%$ of all $t t$ decays. These events are characterized by two high-energy same-sign leptons, two jets (denoted $j$ ) from the hadronization of the $b$-quarks, and large missing energy $\left(\mathbb{E}_{T}\right)$ from two unobserved neutrinos. After lepton and jet reconstruction, we demand that the two hard leptons are of the same sign, a requirement which greatly reduces the SM background, giving rejections of order $10^{-4}$ and $10^{-3}$ for the $t \bar{t}$ and $W W j j$ background processes, respectively. For sextet scalar mesons, after the analysis cuts are imposed, we find 3.2 background events from $t \bar{t}$ and 1.4 from $W W j j$ for $1 \mathrm{fb}^{-1}$ of integrated luminosity. After $b$ tagging and restriction to the $\mu^{+} \mu^{+}$mode, more than half the signal events survive the analysis cuts. In the vector case, after the cuts are imposed, including an asymmetric cut on the momenta of the charged leptons, we find 1.0 background event, 0.4 from $t \bar{t}$ and 0.6 from $W W j j$ for $1 \mathrm{fb}^{-1}$ of integrated luminosity. Again, after $b$-tagging and restriction to the $\mu^{+} \mu^{+}$ mode, $15-30 \%$ of the signal events survive the analysis cuts, depending on the vector mass. Signal and background cross sections are shown in tabular form, before and after cuts, for 6 values of the vector mass in Ref. [⿰] [- and in for 6 values of the scalar mass in Ref. [1].

The search for same-sign top quark pair production in the dilepton mode at the Tevatron imposes an upper limit $\sigma(t t+\bar{t} \bar{t}) \leq 0.7 \mathrm{pb}[3]$. The constraint is plotted in the orange shaded region in Fig. 1. The $t \bar{t}$ invariant mass spectrum in the semi-leptonic decay mode [ $₫$ ] imposes another constraint. Since $b$ and $\bar{b}$ jets from $t \rightarrow W b$ are not distinguished well, $t t$ pairs lead to the same signature as $t \bar{t}$ in the semi-leptonic mode. Hence, the $m_{t \bar{t}}$ spectrum provides an upper limit on $\sigma(t t+\bar{t})$, shown in the cyan shaded region. The lower gray shaded region is the region in which the vector $V$ would hadronize before decay, washing out the spin correlation effects we utilize to probe the coupling and spin of the sextet state.

In Fig. 1, we show the expected numbers of signal events as a function of the vector mass $m_{V}$ for a range of values of the coupling $g_{u u}^{2} \mathrm{Br}(\mathrm{V} \rightarrow \mathrm{tt})$. Based on Poisson statistics, one needs 8 signal events in order to claim a $5 \sigma$ discovery significance on top of 1 background event. We plot the $5 \sigma$ discovery line (solid) in the figure. A corresponding figure for the scalar meson case may be found in Ref. [1]. In the scalar case, the $5 \sigma$ discovery line is found from requiring 14 events.

\section{Top Quark Polarization}

We take the observation of a pair of same-sign dileptons as indicative of a same-sign top quark pair and to suppress SM backgrounds efficiently. The two missing neutrinos in the final state complicate event reconstruction. We use the MT2 method to select the correct $\mu-b$ combinations and to verify whether the final state is consistent with $t \rightarrow W b$ parentage. Then we make use of the on-shell conditions of the two $W$ bosons and two top quarks to solve for the neutrino momenta. Once the neutrino momenta are known, the kinematics of the entire final state is fixed, and the vector boson mass is computed from the invariant mass of the two reconstructed top quarks. The next step is to verify that the top-quarks exhibit opposite polarization, accomplished here by making use of the difference in the momentum spectra of decay leptons from left-handed and right-handed 


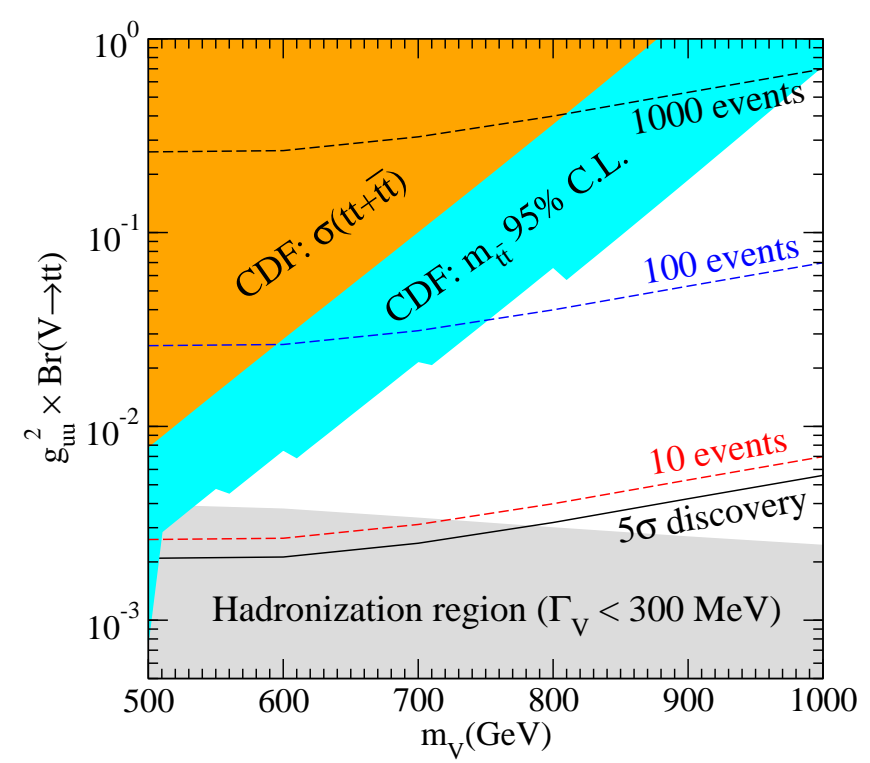

Figure 1: Event number contours as a function of the vector mass and the parameter $g_{u u}^{2} \mathrm{Br}(\mathrm{V} \rightarrow \mathrm{tt})$ after all cuts with an integrated luminosity of $1 \mathrm{fb}^{-1}$. The shaded regions are excluded, as explained in the text.

top quarks. We use the helicity basis to measure the top quark polarization. In this basis, the top quark spin is chosen to be along (against) the direction of motion of the top quark in the center of mass frame of the system. As the charged lepton follows the top quark spin, the lepton from $t_{R}$ decay tends to follow the direction of motion of the top-quark, and it is more energetic. The lepton from the $t_{L}$ decay tends to move against the direction of motion of its top quark, and it is therefore less energetic. In sextet vector boson decay into a $t_{L} t_{R}$ pair, the charged leptons exhibit a mixture of the energetic and soft spectra. To utilize this feature, we order the energy of the leptons and define the top quark containing the greater energy lepton as $t_{\text {greater }}$ and the other top quark as $t_{\text {lesser. }}$ The $\cos \theta$ distributions of the reconstructed $t_{\text {lesser }}$ and $t_{\text {greater }}$ are displayed Ref. [2]. They show that one can differentiate the $(1+\cos \theta)\left(t_{R}\right)$ and $(1-\cos \theta)\left(t_{L}\right)$ shapes.

For a sextet scalar, the expected right-handed polarization can be distinguished from an unpolarized flat distribution at $95 \%$ C.L. with as few as $\mathscr{O}(30)$ events spread over 3 bins in $\cos \theta$, making this measurement possible in with early LHC data; c.f., Fig. 3 in Ref. [1]. In the vector case, 50 to 100 events are needed, spread over 4 bins.

\section{References}

[1] E. L. Berger, Q. H. Cao, C. R. Chen, G. Shaughnessy and H. Zhang, Phys. Rev. Lett. 105 (2010) 181802 [arXiv:1005.2622 [hep-ph]].

[2] H. Zhang, E. L. Berger, Q. H. Cao, C. R. Chen and G. Shaughnessy, Phys. Lett. B 696 (2011) 68 [arXiv:1009.5379 [hep-ph]].

[3] CDF collaboration, T. Aaltonen et al., Phys. Rev. Lett. 102, 041801 (2009), arXiv:0809.4903.

[4] CDF collaboration, T. Aaltonen et al., Phys. Rev. Lett. 102, 222003 (2009), arXiv:0903.2850. 